\title{
Comment on the article by Opeyemi Folorunsho Bamidele, Richard Oluyinka Akintayo, Mathew Olumuyiwa Bojuwoye, et al. "Thrombotic thrombocytopenic purpura as the first presentation in systemic lupus erythematosus"
}

\author{
Maria Maślińska
}

Clinic of Early Arthritis, National Institute of Geriatrics, Rheumatology and Rehabilitation, Warsaw, Poland

The classification criteria for autoimmune diseases are essential for conducting the diagnostic process and eventually establishing a certain diagnosis. However, it is primarily the doctor himself who assesses the clinical picture and results of the performed tests, deciding on the final diagnosis.

The limited availability of some of the diagnostic methods, including laboratory tests, may restrict the scope of the performed analysis - as in the case of the present article, whose authors were limited by the financial resources of the research center and of the patient.

According to the previous classification criteria of systemic lupus erythematosus (SLE), establishing the diagnosis depended on whether the patient presented a minimal number of characteristic symptoms [1]. The new criteria, which should be formally approved by the end of 2018 [2], apply a point system that assigns a different weight to each of the 22 criteria. Obtaining more than 10 points in such an assessment will be equivalent to the SLE diagnosis, provided that the initial requirement of the presence of antinuclear antibodies (ANA) in a 1: 80 titer tested with Hep-2 cells or established with another equivalent test - is met.

The described case may be considered as a starting point for a discussion on SLE diagnostic criteria, with the described coexistence of TTP and SLE being particularly interesting.

\section{References}

1. Petri M, Orbai AM, Alarcon GS, et al. Derivation and validation of the Systemic Lupus International Collaborating Clinics classification criteria for systemic lupus erythematosus. Arthritis Rheum 2012; 64: 2677-2686.

2. Aringer M, Costenbader K, Brinks R, et al. OP0020 Validation of new systemic lupus erythematosus classification criteria. Ann Rheum Dis 2018; 77: 60 